\title{
Review Article \\ New Oral Anticoagulants for Thromboprophylaxis after Elective Total Hip and Knee Arthroplasty
}

\author{
Richard J. Friedman \\ Department of Orthopaedic Surgery, Roper Hospital, Charleston Orthopaedic Associates, \\ 1012 Physicians Drive, Charleston, SC 29414, USA \\ Correspondence should be addressed to Richard J. Friedman, rjfriedman@mybones.com \\ Received 16 March 2010; Revised 28 May 2010; Accepted 6 June 2010 \\ Academic Editor: Gualtiero Palareti \\ Copyright ( 92010 Richard J. Friedman. This is an open access article distributed under the Creative Commons Attribution \\ License, which permits unrestricted use, distribution, and reproduction in any medium, provided the original work is properly \\ cited.

\begin{abstract}
Anticoagulant drugs reduce the risk of venous thromboembolic events after total hip and knee arthroplasty. However, the use of current drugs, such as low molecular weight heparins, is hampered by their subcutaneous route of administration. The use of vitamin $\mathrm{K}$ antagonists is hampered by the requirement for routine coagulation monitoring and dose titration to provide effective anticoagulation without an increased risk of bleeding and numerous food and drug interactions. Clearly, there is a need for new oral, fixed-dose anticoagulant drugs that do not require coagulation monitoring, while demonstrating similar or better
\end{abstract} \\ efficacy and safety profiles when compared with current agents.
}

\section{Introduction}

In 2007, the annual number of total hip and knee arthroplasties (THAs and TKAs) in the US was 250,000 and 500,000 , respectively [1]. These numbers are expected to increase to 572,000 and 3.48 million for primary THA and TKA, respectively, between 2005 and 2030 [1]. Orthopaedic surgeons and internists are fully aware of these expected increases in the number of elective THAs/TKAs.

The types of patients undergoing THA/TKA are consistent and the risks of surgery are well characterized. Antibiotic prophylaxis for THA/TKA is estimated to decrease the relative risk of wound infection by $81 \%$ compared with no prophylaxis [2]. Similarly, the appropriate use of anticoagulant drugs has been shown to reduce the risk of venous thromboembolism (VTE) after THA/TKA, and guidelines recommend their routine use after this type of surgery. Without prophylaxis, the incidence of venographic deep vein thrombosis (DVT) and of pulmonary embolism (PE) after THA are $42-57 \%$ and $0.9-28 \%$, respectively [3]. The index event usually occurs at a mean of 21.5 (standard deviation 22.5) days after surgery-typically after hospital discharge [4]. The risk of venographic DVT and PE after TKA is $41-85 \%$ and $1.5-10 \%$, respectively [3]. Clinical symptomatic events usually occur at a mean of 9.7 days after TKA and 21.5 days after THA [4], with 75\% occurring after a median hospital stay of 5 days for THA. The current trend is towards much shorter hospital stays, with a mean of less than 3 days for THA and TKA at Roper Hospital (Charleston, SC, USA) in 2009, meaning that the vast majority of symptomatic events will occur on an outpatient basis and, therefore, prophylaxis is mainly an outpatient issue.

The American College of Chest Physicians (ACCP) guidelines recommend prophylaxis with anticoagulants for a minimum of 10 days and up to 35 days after THA to reduce the risk of VTE (Grade 1A). After TKA, the ACCP recommends prophylaxis with anticoagulants for at least 10 days (Grade 1A) and suggests up to 35 days in some patients (Grade 2B) [3]. Options include vitamin $\mathrm{K}$ antagonists (VKAs), such as warfarin, low molecular weight heparins (LMWHs), such as enoxaparin, and the synthetic pentasaccharide fondaparinux. Although the antiplatelet acetylsalicylic acid (ASA) is considered by some clinicians to have a role in the prevention of $\mathrm{PE}$, its use alone for thromboprophylaxis is not recommended by the ACCP.

The American Academy of Orthopaedic Surgeons (AAOS) has published guidelines strictly on the prevention 
of PE, not DVT prophylaxis, recommending that patients at standard risk of both $\mathrm{PE}$ and major bleeding should be considered for one of the prophylactic agents evaluated in their guideline, including ASA, LMWHs, synthetic pentasaccharides and warfarin. Those at increased (above standard) risk of PE and standard risk of major bleeding should be considered for one of the prophylactic agents evaluated in their guideline, including LMWHs, synthetic pentasaccharides, and warfarin. Patients at standard risk of $\mathrm{PE}$ and at increased risk of major bleeding should be considered for prophylaxis with ASA or warfarin, as evaluated in their guideline [5]. However, they fail to provide any definitions or guidelines regarding what patients are at increased risk of bleeding and increased risk of PE, or the standard risk of bleeding and PE.

Although the AAOS does not specifically give guidance on the prevention of DVT after THA/TKA, DVT prophylaxis is as important as the prevention of $\mathrm{PE}$ because after an initial DVT (any cause), patients have a $10 \%$ risk of recurrent VTE after 1 year [6]. The risk of recurrence is $\leq 3 \%$ per year in patients with transient risk factors (such as recent surgery) [7]. Following an episode of DVT, there is an approximate $24 \%$ risk of postthrombotic syndrome after 3 years [6]. Of all untreated initial calf vein thrombi (distal DVT), 20\% extend proximally [8]. Moreover, thrombus resolution is slower and postthrombotic syndrome is more severe after proximal than distal DVT [9].

The clinical challenges that orthopaedic surgeons, internists, and clinicians face are that current anticoagulants are administered subcutaneously or require monitoring and dose titration to provide effective anticoagulation without increasing bleeding risk. More effective and convenient alternative anticoagulants, which can be given at fixed doses without routine coagulation monitoring, could improve current clinical practice $[10,11]$. New oral anticoagulant drugs are being developed that address these issues, while having similar or better efficacy and safety profiles when compared with current agents.

This paper will review the unmet clinical needs with current agents, discuss the new classes of oral agents, present data on the new oral agents currently available in the European Union (EU) and other countries, and discuss how these agents might meet the needs of orthopaedic surgeons and internists in VTE prophylaxis.

\section{Current Anticoagulant Regimens}

2.1. Parenteral Anticoagulants. Although unfractionated heparins (UFHs) have been available since the early 1930s, studies in the 1970s demonstrated that they prevented VTE and fatal PE in patients undergoing surgery $[12,13]$. UFHs act at several points of the coagulation cascade (Figure 1). Parenteral LMWHs, which emerged in the early 1980s, also act at several levels of the coagulation cascade [14] (Figure 1).

During the 1990s, a comprehensive series of studies demonstrated the clinical value of LMWHs in reducing the risk of VTE [15] (Table 1). Compared with UFHs, LMWHs offered a convenient solution-they were available as fixed doses, did not require routine coagulation monitoring or dose adjustment [14], and led to clinically significant reductions in the number of venous thromboembolic events [15]. The different LMWHs are created chemically or by depolymerization of UFH. LMWHs target both Factor Xa and Factor IIa (thrombin) [14]. The ratio of Factor Xa : Factor IIa inhibition differs between the different available LMWHs and these ratios are considered to be related to safety and efficacy (the greater the level of Factor Xa inhibition, the greater the efficacy [16] because Factor Xa is generated earlier in the coagulation cascade than thrombin). The ratio of Factor Xa : Factor IIa inhibition ranges from 2:1 to $4: 1$ for the different LMWHs in current use, compared with $1: 1$ for UFH [14], indicating that antithrombotic activity may be higher when using LMWHs, without the increased risk of bleeding.

Fondaparinux (2.5 mg once daily), a subcutaneously administered, indirect Factor Xa inhibitor (Figure 1), was more effective than enoxaparin (30 mg every 12 hours after TKA and $40 \mathrm{mg}$ once daily after THA) in reducing the risk of VTE [17, 18]. The timing of fondaparinux administration affected the efficacy and incidence of bleeding events after THA/TKA: major bleeding was significantly higher in patients who received their first dose $<6$ hours after skin closure $(3.2 \%)$ than in those where the first dose was delayed to $\geq 6$ hours $(2.1 \%, P=.045)$. This effect was more evident in patients who weighed $<50 \mathrm{~kg}$, those $>75$ years of age, and those with moderate renal impairment [19].

It is important to note that bleeding events are always likely after surgery-affecting approximately $2.4 \%$ of patients even when no anticoagulants are used [20] - and anticoagulants do not increase bleeding risk when administered correctly with regards to dosage, timing and concomitant use of other agents that affect bleeding (such as nonsteroidal anti-inflammatory drugs). LMWHs offer a good balance, by reducing the number of venous thromboembolic events while maintaining low bleeding rates. However, recent studies have highlighted that only approximately half of patients in the US receive prophylaxis after THA/TKA at the timing, duration and intensity recommended by the ACCP $[3,21]$. Worldwide, $59 \%$ of surgical patients (undergoing any type of surgery) at risk of VTE receive ACCP-recommended prophylaxis [22]. Furthermore, the duration of prophylaxis is often shorter than the period in which thromboembolic events occur after surgery [4]. Possible reasons for this are that surgeons may not be aware of the substantial postdischarge risk of thromboembolic events, cost, lack of convenience, and need for monitoring.

2.2. Oral Antithrombotics. Developed in the 1950s, the VKAs, such as warfarin, indirectly inhibit the production of several coagulation factors (Figure 1). Although recommended in the ACCP guidelines, studies have shown that warfarin is not as effective as parenteral anticoagulants in reducing the venographic DVT incidence [15] (Table 1). Although it is an oral agent, warfarin is less convenient than parenteral anticoagulants, mainly due to the need for frequent monitoring and dose adjustments, and food and drug interactions. Owing to its slow onset of action, it can take 2-4 days for a therapeutic 

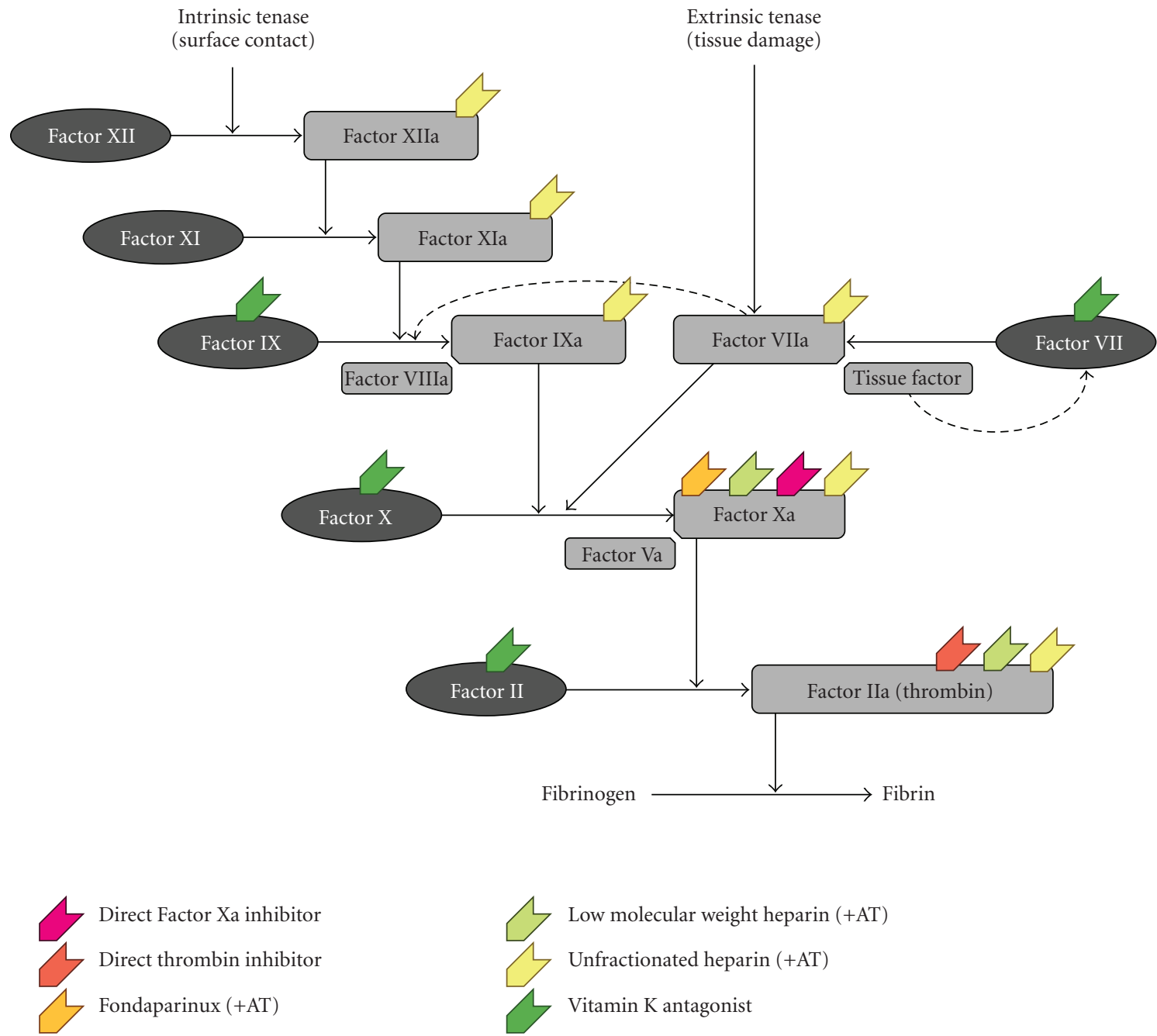

FIgURE 1: Coagulation cascade: where anticoagulants act. AT, antithrombin.

TABLE 1: Deep vein thrombosis frequencies following prophylaxis with different agents after total hip and knee arthroplasties [15].

\begin{tabular}{|c|c|c|c|c|}
\hline & \multicolumn{2}{|c|}{ DVT frequency after total hip arthroplasty } & \multicolumn{2}{|c|}{ DVT frequency after total knee arthroplasty } \\
\hline & Prevalence $(\%)$ & Relative risk reduction (\%) & Prevalence $(\%)$ & Relative risk reduction (\%) \\
\hline Acetylsalicylic acid & 40.2 & 26 & 56.0 & 13 \\
\hline Low molecular weight heparins & 16.1 & 70 & 30.6 & 52 \\
\hline Warfarin & 22.1 & 59 & 46.8 & 27 \\
\hline
\end{tabular}

DVT, deep vein thrombosis.

international normalized ratio (INR; range: $2-3$ [3]) to be reached. Warfarin has an unpredictable pharmacological profile and dosing needs to be individualized. With a narrow window for safety and efficacy, coagulation monitoring is essential to ensure that patients remain within the INR range after discharge; patients have to be taught how to monitor their INR and take the correct dose at home or frequently attend clinics or a primary care physician. Furthermore, warfarin has many food and drug interactions that may potentiate or inhibit its action, which may be problematic in patients taking concomitant medications for comorbid conditions [23].
A recent study showed that although pharmacy acquisition costs of warfarin are lower than subcutaneous anticoagulant drugs, the total 6-month costs were lower with subcutaneous anticoagulant drugs. Therefore, the initial savings may be offset by a higher incidence of venous thromboembolic events and higher 6-month medical costs with warfarin [24].

The use of ASA remains controversial. It is important to note that ASA is an antiplatelet and not an anticoagulant, but some clinicians consider it to have a role in the prevention of fatal PE and its use is recommended by the AAOS [5] for the prevention of PE only, not for DVT. They recommend 
that for patients at standard risk of both PE and major bleeding, who represent the majority of patients undergoing total joint arthroplasty, ASA (325 mg twice daily, or $81 \mathrm{mg}$ once daily if gastrointestinal symptoms develop, for 6 weeks) may be one of the prophylactic drugs considered, along with warfarin, $\mathrm{LMWH}$, and fondaparinux. The guidelines do not address other venous thromboembolic events, such as DVT, and do not define standard or increased risk of bleeding or PE. ASA has been shown to reduce venous thromboembolic events by $26 \%$ and $13 \%$ (relative risk reductions) in patients undergoing THA and TKA, respectively (Table 1) [15], which is less than the reduction with other prophylactic agents.

2.3. New Oral Anticoagulants. The ideal anticoagulant needs to be more effective without increasing bleeding risk, safe, convenient to use, administered orally once daily and have fixed dosing-factors that could potentially improve patient compliance. The most promising new oral anticoagulants are the direct thrombin inhibitors (such as dabigatran) and the direct Factor Xa inhibitors (such as rivaroxaban and apixaban) - agents that directly target a single coagulation factor in the coagulation cascade (Figure 1). Dabigatran is approved in the EU and Canada (110 mg within 1-4 hours of surgery, then $220 \mathrm{mg}$ once daily for 28-35 days after THA and 10 days after TKA) for VTE prophylaxis after elective THA/TKA in adults [25]. Rivaroxaban is approved in the EU and numerous other countries for the prevention of VTE in adult patients after elective hip or knee arthroplasty (10 mg 6-10 hours after surgery, then once daily for 35 days after THA and 14 days after TKA [26]). These two drugs represent the first new oral agents for VTE prophylaxis in THA and TKA in over 50 years.

2.3.1. Apixaban. Apixaban is an oral, direct Factor Xa inhibitor with predictable pharmacokinetics and pharmacodynamics [27]. Gender has no clinically relevant effect on apixaban [28]. Data are lacking for the effects of body weight or old age on apixaban. Approximately half of administered apixaban is absorbed and half is recovered in faeces. Of the total dose, approximately one-third is recovered in urine, of which over $80 \%$ is apixaban [29].

Digoxin and inhibitors or substrates of P450 enzymes do not have clinically relevant interactions with apixaban $[30,31]$. Absorption of apixaban is not affected after a highcalorie meal [32].

A phase II study [33] of apixaban was used to establish the dose to be used for the phase III clinical development programme. In this study, 1,238 patients were randomized to one of six double-blind apixaban doses $(5,10$ or $20 \mathrm{mg}$ once daily or $2.5,5$ or $10 \mathrm{mg}$ twice daily), enoxaparin (30 mg twice daily) or open-label warfarin (titrated to an INR of 1.8-3.0), for 10-14 days. The primary efficacy outcome (the composite of VTE and all-cause mortality) decreased with increasing apixaban dose $(P=.09$ for once-daily or twicedaily regimens combined, $P=.19$ for once-daily and $P=.13$ for twice-daily dosing). There was a significant dose-related increase of total adjudicated bleeding events for the oncedaily $(P=.01)$ and twice-daily $(P=.02)$ regimens. The authors concluded that apixaban $2.5 \mathrm{mg}$ twice daily and 5 mg once daily might have a promising risk-benefit profile compared with enoxaparin $30 \mathrm{mg}$ twice daily and warfarin.

The ADVANCE-1 phase III study compared apixaban $2.5 \mathrm{mg}$ twice daily with the enoxaparin regimen commonly used in North America of $30 \mathrm{mg}$ twice daily, for the prevention of VTE after TKA $(N=3,195)$ [34]. The primary efficacy outcome (composite of venographic DVT, symptomatic, objectively confirmed DVT or nonfatal PE, or death from any cause) occurred in $9.0 \%$ of patients receiving apixaban and $8.8 \%$ of patients receiving enoxaparin (relative risk $1.02,95 \%$ confidence interval $[\mathrm{CI}] 0.78$ to $1.32, P=.06$ for noninferiority, statistical criteria not met) during the treatment period. The rates of PE were $1.0 \%$ in the apixaban group and $0.4 \%$ in the enoxaparin group; two PEs were fatal in the apixaban group and none were fatal in the enoxaparin group. Major or clinically relevant nonmajor bleeding occurred in $2.9 \%$ and $4.3 \%$ of patients receiving apixaban and enoxaparin, respectively $(P=.03)$. Major bleeding occurred in $0.7 \%$ and $1.4 \%$ of patients receiving apixaban and enoxaparin, respectively $(P=.053)$. One patient in the enoxaparin group died from bleeding; none of the apixaban group died from bleeding. In the ADVANCE2 study, which compared apixaban $2.5 \mathrm{mg}$ twice daily with enoxaparin $40 \mathrm{mg}$ once daily (also in patients undergoing TKA; $N=3,057$ ), the hypothesis was that apixaban would be noninferior to enoxaparin based on a prespecified margin for the primary efficacy outcome in which the upper limit of the two-sided $95 \% \mathrm{CI}$ is $<1.25$ for relative risk and $<5.6 \%$ for the absolute risk difference [35]. If both criteria were met, superiority was tested. The primary efficacy endpoint (the same as in ADVANCE-1) occurred in $15.1 \%$ of the apixaban group and $24.4 \%$ of the enoxaparin group (relative risk 0.62 , 95\% CI 0.51 to $0.74, P<.0001$ for noninferiority and superiority; absolute risk difference $-9.3 \%, 95 \% \mathrm{CI}-12.7$ to $-5.8, P<.0001$ for noninferiority). Two patients receiving apixaban died from $\mathrm{PE}$ and one patient receiving enoxaparin died from bleeding. Major or clinically relevant nonmajor bleeding occurred in $3.5 \%$ of the apixaban group and $4.8 \%$ of the enoxaparin group $(P=.09)$. In summary, the findings of these studies suggest that apixaban is significantly more effective than the $40 \mathrm{mg}$ once-daily enoxaparin regimen at reducing the composite of DVT, PE and death by any cause, with no increased risk of major bleeding. In ADVANCE-1, apixaban did not meet the prespecified statistical criteria for noninferiority of efficacy compared with enoxaparin $30 \mathrm{mg}$ twice daily.

2.3.2. Dabigatran Etexilate. Dabigatran is an oral, oncedaily, direct thrombin inhibitor that can be given in a fixed oral dose without dose adjustment for age, body weight or gender [36, 37]. It has a rapid onset of action and provides predictable anticoagulation without the need for routine coagulation monitoring [36]. The main elimination pathway is renal excretion, accounting for more than $80 \%$ of the systemically available dose of dabigatran [38].

Therapeutic doses of dabigatran are unlikely to interact with drugs that are metabolized by the CYP450 system [38]. It has been shown that food delays the time to peak plasma 
concentration by 2 hours, but does not have a relevant effect on the extent of dabigatran absorption [39].

Dose-ranging studies in patients undergoing THA suggested that the therapeutic window was $12.5-300 \mathrm{mg}$ twice daily (BISTRO I [40]) and in patients undergoing THA and TKA the optimal total daily dose was 100-300 mg (BISTRO II [41]).

Two phase III, randomized trials in patients undergoing TKA have been conducted, one with most of its participating centres in the EU and one in North America, comparing dabigatran with enoxaparin. In the European study (REMODEL; $N=2,101[42,43]$ ), once-daily dabigatran (first dose was half of the subsequent dose; subsequent doses were $150 \mathrm{mg}$ and $220 \mathrm{mg}$ in two tablets) was as effective (noninferior) as once-daily enoxaparin ( $40 \mathrm{mg}$ ) for preventing VTE and all-cause mortality in patients undergoing TKA $(40.5 \%$ of the dabigatran $150 \mathrm{mg}$ group [absolute risk difference versus enoxaparin $2.8 \%$ ], 36.4\% of the dabigatran $220 \mathrm{mg}$ group [absolute risk difference versus enoxaparin $-1.3 \%$ ] and $37.7 \%$ of the enoxaparin group), with similar bleeding rates (compared with enoxaparin: $P=1.0$ for the dabigatran $150 \mathrm{mg}$ dose and $P=.082$ for the dabigatran $220 \mathrm{mg}$ dose). However, in the RE-MOBILIZE study $(N=2,615)[43,44]$, which used the usual North American enoxaparin regimen of $30 \mathrm{mg}$ twice daily, dabigatran $150 \mathrm{mg}$ and $220 \mathrm{mg}$ showed inferior efficacy to enoxaparin for the primary outcome of total VTE and death $(33.7 \%$ of the $150 \mathrm{mg}$ group [absolute risk difference versus enoxaparin $8.4 \%, P=.0009$ for noninferiority versus enoxaparin], $31.1 \%$ of the dabigatran $220 \mathrm{mg}$ group [absolute risk difference versus enoxaparin $5.8 \%, P=.0234]$ and $25.3 \%$ of the enoxaparin group), although bleeding rates were similar between all three groups ( $0.6 \%$ of the $150 \mathrm{mg}$ group, $0.6 \%$ of the $220 \mathrm{mg}$ group and $1.4 \%$ of the enoxaparin group). The secondary outcome of major VTE (defined as proximal DVT, PE and VTE-related death) occurred in $3.0 \%$ of the dabigatran $150 \mathrm{mg}$ group (risk difference with enoxaparin $0.8 \%, P=.36$ ), $3.4 \%$ of the dabigatran $220 \mathrm{mg}$ group (risk difference with enoxaparin $1.2 \%, P=.21$ ) and $2.2 \%$ of the enoxaparin group.

The RE-NOVATE study compared once-daily dabigatran $220 \mathrm{mg}$ or $150 \mathrm{mg}$ with once-daily enoxaparin $40 \mathrm{mg}$ after THA $(N=3,494)$. Both doses of dabigatran were noninferior to enoxaparin for the composite of total VTE and death (8.6\% of the $150 \mathrm{mg}$ group [absolute risk difference versus enoxaparin $1.9 \%, P<.0001$ for noninferiority versus enoxaparin], $6.0 \%$ of the $220 \mathrm{mg}$ group [absolute risk difference versus enoxaparin $-0.7 \%, P<.0001$ for noninferiority versus enoxaparin] and $6.7 \%$ of the enoxaparin group). Rates of major bleeding did not differ significantly between the groups $(1.3 \%$ for the $150 \mathrm{mg}$ group $[P=.60], 2.0 \%$ for the $220 \mathrm{mg}$ group $[P=.44]$ and $1.6 \%$ for the enoxaparin group). There were no significant differences in cardiac events or liver enzyme elevations in any of the three groups $[43,45]$. Whereas RE-MODEL and RE-NOVATE showed the tested doses of dabigatran were noninferior to the 40-mg enoxaparin regimen for VTE prophylaxis, RE-MOBILIZE found dabigatran to be inferior to the 30-mg twice-daily enoxaparin regimen. Possible reasons for this finding are the higher daily dosage of enoxaparin and longer treatment duration in the RE-MOBILIZE study compared with the REMODEL study.

A meta-analysis of the three dabigatran studies (which assessed the recommended $220 \mathrm{mg}$ dose) supported the findings of RE-MODEL and RE-NOVATE [46]. It showed that there were no significant differences between dabigatran $220 \mathrm{mg}$ and enoxaparin in any endpoints when RE-MODEL and RE-NOVATE were analysed $(P>.15)$, or when all three trials were included in the analysis (all $P>.30$ ). Risk ratios (random effects) for the composite of total VTE and allcause mortality were 0.95 (95\% CI 0.82 to 1.10 ) in the twotrial analysis and 1.05 (95\% CI 0.87 to 1.26$)$ in the threetrial analysis. Major bleeding rates did not differ significantly when RE-MODEL and RE-NOVATE were analysed $(P=.41$ for random-effects and fixed-effects analyses) or when all three studies were analysed ( $P=.85$ for random-effects and $P=.95$ for fixed-effects analyses).

In a recent prespecified pooled analysis of the studies, the primary outcome (composite outcome of major and VTErelated mortality) occurred in $3.3 \%$ of the enoxaparin group, $3.8 \%$ of the $150 \mathrm{mg}$ group (risk difference $0.5 \%, 95 \% \mathrm{CI}-0.6$ to $1.6, \mathrm{I}^{2}=0 \%$ [if $\mathrm{I}^{2}$ was greater than $50 \%$, heterogeneity was considered to be substantial]) and $3.0 \%$ of the dabigatran $220 \mathrm{mg}$ group (risk difference versus enoxaparin $-0.2 \%, 95 \%$ $\mathrm{CI}-1.3$ to $\left.0.9, \mathrm{I}^{2}=37 \%\right)$. Rates of major bleeding were $1.4 \%$ in the enoxaparin group, $1.1 \%$ in the $150 \mathrm{mg}$ group (risk difference $-0.4 \%, 95 \% \mathrm{CI}-1.0$ to $\left.0.2, \mathrm{I}^{2}=0 \%\right)$ and $1.4 \%$ in the dabigatran $220 \mathrm{mg}$ group (risk difference $-0.2 \%, 95 \% \mathrm{CI}$ -0.8 to $0.5, \mathrm{I}^{2}=40 \%$ ). These findings suggest that dabigatran was as effective as enoxaparin and the risk of major bleeding was similar [47].

2.3.3. Rivaroxaban. Rivaroxaban-an oral, direct Factor Xa inhibitor-was found to exhibit a predictable pharmacokinetic and pharmacodynamic profile and does not require dose adjustment for age, gender [48] or weight [49]. Rivaroxaban and its metabolites have a dual route of elimination: one-third of the administered drug is cleared as unchanged active drug by the kidneys; one-third is metabolized to inactive metabolites and then excreted by the kidneys; and one-third is metabolized to inactive metabolites and then excreted by the faecal route [50].

Rivaroxaban has a low propensity for drug-drug interactions with frequently used concomitant medications, such as naproxen [51], ASA [52] or clopidogrel [53], and no interaction with the cardiac glycoside digoxin [54]. Dietary restrictions are not necessary and rivaroxaban was given with or without food in the phase III VTE prevention studies (RECORD1-4 [REgulation of Coagulation in ORthopaedic surgery to prevent Deep vein thrombosis and pulmonary embolism]).

Phase II studies [55-58] showed that all investigated rivaroxaban dose regimens had similar efficacy to enoxaparin, and the incidence of major bleeding was not significantly different to enoxaparin across a fourfold dose range (5-20 mg total daily rivaroxaban dose).

The RECORD programme comprised four phase III studies investigating the efficacy and safety of rivaroxaban in 12,500 patients undergoing THA and TKA [59-62]. All 
patients received rivaroxaban $10 \mathrm{mg}$ once daily $6-8$ hours after surgery, and there was no upper age or weight limit for participation. The primary efficacy endpoint was the composite of DVT, nonfatal PE and all-cause mortality up to day 30-42 after surgery for RECORD1 and RECORD2, up to day 13-17 for RECORD3 and up to day 17 for RECORD4. The main safety endpoint was the incidence of treatment-emergent (observed no later than 2 days after the last dose of the study drug) major bleeding events. Other safety outcomes (for example, nonmajor bleeding and postoperative wound infection) were also reported [59-62].

RECORD1 showed that 5 weeks of extended-duration rivaroxaban (10 mg once daily for 31-39 days after surgery) was significantly more effective than enoxaparin ( $40 \mathrm{mg}$ once daily for 31-39 days) for extended-duration prophylaxis in patients undergoing THA (1.1\% versus $3.7 \%$ for the primary efficacy endpoint, $P<.001$ ) [59]. Major bleeding events did not differ significantly between the groups $(0.3 \%$ versus $0.1 \%$ of patients, $P=.18$ ). Clinically relevant nonmajor bleeding occurred in $2.9 \%$ of the rivaroxaban group versus $2.4 \%$ of the enoxaparin group; haemorrhagic wound complications in $1.5 \%$ versus $1.7 \%$ of patients; and postoperative wound infections in $0.4 \%$ of patients in both groups. The incidence of symptomatic VTE during treatment was not significantly different between the groups ( $0.3 \%$ versus $0.5 \%, P=.22)$.

RECORD2 demonstrated that extended-duration rivaroxaban prophylaxis (10 mg once daily for 31-39 days after surgery) was significantly more effective than short-duration prophylaxis with enoxaparin $(40 \mathrm{mg}$ once daily for 10-14 days) followed by placebo in patients undergoing THA (2.0\% versus $9.3 \%$ for the primary efficacy endpoint, $P<.0001$ ) [60]. The incidence of bleeding was comparable between extended-regimen rivaroxaban and short-duration enoxaparin. Major bleeding events occurred in $<0.1 \%$ of patients in both groups. Clinically relevant nonmajor bleeding was recorded in $3.3 \%$ of the rivaroxaban group versus $2.7 \%$ of the enoxaparin group; haemorrhagic wound complications in $1.6 \%$ versus $1.7 \%$ of patients; and postoperative wound infections in $0.7 \%$ versus $0.5 \%$ of patients, respectively. Significantly fewer patients in the rivaroxaban group had symptomatic VTE $(0.2 \%)$ than in the enoxaparin group $(1.2 \%, P=.004)$ during the active study period.

In RECORD3, rivaroxaban prophylaxis (10 mg once daily for 10-14 days) was significantly more effective than the European enoxaparin regimen for prophylaxis $(40 \mathrm{mg}$ once daily) in patients undergoing TKA (9.6\% versus $18.9 \%$ for the primary efficacy endpoint, $P<.001$ ), with a similar safety profile [61]. Rates of major bleeding were similar in the rivaroxaban and enoxaparin groups $(0.6 \%$ versus $0.5 \%, P=$ .77); clinically relevant nonmajor bleeding occurred in $2.7 \%$ versus $2.3 \%$ of patients; haemorrhagic wound complications in $2.0 \%$ versus $1.9 \%$ of patients; and postoperative wound infections in $0.6 \%$ versus $0.9 \%$ of patients. There was a significant reduction in the number of symptomatic venous thromboembolic events in the rivaroxaban group $(0.7 \%$ versus $2.0 \%, P=.005)$.

In RECORD4, rivaroxaban showed significantly better efficacy than the enoxaparin regimen $(30 \mathrm{mg}$ every 12 hours) commonly used in North America for short-term prophylaxis after TKA (6.9\% versus $10.1 \%$, respectively, for the primary efficacy endpoint, $P=.0118)$ [62]. The rates of major bleeding were $0.7 \%$ versus $0.3 \%(P=.1096)$, respectively; clinically relevant nonmajor bleeding occurred in $2.6 \%$ versus $2.0 \%$ of patients; haemorrhagic wound complications in $1.4 \%$ versus $1.5 \%$ of patients; and postoperative wound infections in $0.3 \%$ versus $0.2 \%$ of patients, respectively. The observed incidences of symptomatic VTE in those receiving rivaroxaban or enoxaparin were $0.7 \%$ versus $1.2 \%(P=.187)$, respectively.

In the four studies comparing rivaroxaban with enoxaparin, rivaroxaban demonstrated superior efficacy compared with enoxaparin. In addition, extended thromboprophylaxis with rivaroxaban was significantly more effective than shortterm enoxaparin plus placebo in the prevention of total, major and symptomatic VTE after THA. Furthermore, the incidence of treatment-emergent major and clinically relevant nonmajor bleeding was low for rivaroxaban and enoxaparin $(P=.21$ [data on file] for RECORD1, $P=.39$ [data on file] for RECORD2, $P=.44$ for RECORD3 [61] and $P=.18$ for RECORD4 [62]). There was no evidence of compromised liver function or rebound cardiovascular events associated with rivaroxaban.

In a pooled analysis of the RECORD1, 2 and 3 studies (which compared rivaroxaban with enoxaparin $40 \mathrm{mg}$ once daily after THA and TKA) [63], the prespecified primary efficacy outcome (the composite of symptomatic VTE [DVT or PE] and all-cause mortality at 2 weeks) was $0.4 \%$ and $0.8 \%$, respectively $(P=.005)$. The rates were $0.5 \%$ and $1.3 \%$, respectively, at the end of the planned medication period $(P<.001)$. Rates of on-treatment major bleeding were $0.2 \%$ for both drugs at 2 weeks $(P=.662)$, and $0.3 \%$ for rivaroxaban and $0.2 \%$ for enoxaparin at the end of the planned medication period $(P=.305)$. Rates of clinically relevant nonmajor bleeding were $2.6 \%$ for rivaroxaban and $2.3 \%$ for enoxaparin at 2 weeks, and $3.0 \%$ and $2.5 \%$, respectively, at the end of the planned medication period $(P-$ values not reported).

In a pooled analysis of all four RECORD studies [64], the primary efficacy endpoint (the composite of symptomatic VTE [DVT or PE] and death) was significantly reduced for the rivaroxaban regimens compared with enoxaparin regimens at day $12 \pm 2(0.5 \%$ versus $1.0 \%, P=.001)$, in the planned treatment period $(0.6 \%$ versus $1.3 \%, P<.001)$, and in a post hoc analysis of the treatment and follow-up period $(0.8 \%$ versus $1.6 \%, P<.001)$. Rates of treatmentemergent major bleeding were not significantly different between groups at any of the time points analysed [64]. The composite of major and clinically relevant nonmajor bleeding did not differ at day $12 \pm 2(P=.186)$, but was significantly higher for rivaroxaban in the planned medication period $(P=.039)$. Rates of the composite of $\mathrm{PE}$ and death were lower for rivaroxaban compared with enoxaparin in the planned treatment period and follow-up (0.5\% versus $0.8 \%, P=.039)$ [65].

Future research needs to assess whether changing the timing of the first dose could improve the safety profile without significantly affecting efficacy. In theory, the earlier 
an anticoagulant is given, the better the efficacy, but at a cost of increased bleeding [66]. Conversely, the longer anticoagulation is delayed, the lower the risk of bleeding, but efficacy may decrease too.

\section{Summary and Conclusions}

Among the numerous oral anticoagulants currently in phase II and III development, three of the oral agents-apixaban, dabigatran and rivaroxaban-hold considerable potential benefits for improving thromboprophylaxis strategies. In light of recent promising findings, more studies on direct thrombin inhibitors and Factor Xa inhibitors are likely. In addition, reports from daily clinical practice will indicate whether the new agents will change current practice [67]. A phase III TKA study has shown that apixaban is significantly more effective than the once-daily enoxaparin regimen, without an increase in bleeding. The phase III studies comparing dabigatran with enoxaparin were designed to show the noninferiority of dabigatran. It was found that dabigatran has similar efficacy and safety compared with the once-daily enoxaparin regimen in THA and TKA. In addition, phase III studies have shown significantly improved efficacy and similar safety for rivaroxaban compared with both once-daily and twice-daily enoxaparin regimens in THA and TKA. All of these agents provide the benefit of oral dosing without the need for monitoring or dose adjustment, thereby improving the convenience of prophylaxis.

\section{Disclosures}

The author has received research or institutional support from Boehringer Ingelheim and Astellas US and has been a paid consultant for Astellas US, Boehringer Ingelheim, Johnson \& Johnson, and DJO Surgical.

\section{Acknowledgments}

The author would like to acknowledge Li Wan who provided medical writing services with funding from Bayer Schering Pharma AG and Johnson \& Johnson Pharmaceutical Research \& Development, L.L.C.

\section{References}

[1] S. Kurtz, K. Ong, E. Lau, F. Mowat, and M. Halpern, "Projections of primary and revision hip and knee arthroplasty in the United States from 2005 to 2030," Journal of Bone and Joint Surgery American, vol. 89, no. 4, pp. 780-785, 2007.

[2] B. AlBuhairn, D. Hind, and A. Hutchinson, "Antibiotic prophylaxis for wound infections in total joint arthroplasty : a systematic review," Journal of Bone and Joint Surgery British, vol. 90, no. 7, pp. 915-919, 2008.

[3] W. H. Geerts, D. Bergqvist, G. F. Pineo et al., "Prevention of venous thromboembolism: American College of Chest Physicians evidence-based clinical practice guidelines (8th edition)," Chest, vol. 133, no. 6, supplement, pp. 381S-453S, 2008.
[4] D. Warwick, R. J. Friedman, G. Agnelli et al., "Insufficient duration of venous thromboembolism prophylaxis after total hip or knee replacement when compared with the time course of thromboembolic events," Journal of Bone and Joint Surgery British, vol. 89, no. 6, pp. 799-807, 2007.

[5] American Academy of Orthopaedic Surgeons, "American Academy of Orthopaedic Surgeons clinical guideline on prevention of symptomatic pulmonary embolism in patients undergoing total hip or knee arthroplasty," 2007, http://www.aaos.org/Research/guidelines/PE_guideline.pdf.

[6] P. Prandoni, A. W. A. Lensing, A. Cogo et al., "The long-term clinical course of acute deep venous thrombosis," Annals of Internal Medicine, vol. 125, no. 1, pp. 1-7, 1996.

[7] C. Kearon, "Natural history of venous thromboembolism," Circulation, vol. 107, no. 23, supplement, pp. I22-I30, 2003.

[8] W. P. Gorman, K. R. Davis, and R. Donnelly, "ABC of arterial and venous disease. Swollen lower limb-1: general assessment and deep vein thrombosis," British Medical Journal, vol. 320, no. 7247, pp. 1453-1456, 2000.

[9] A. M. Asbeutah, A. Z. Riha, J. D. Cameron, and B. P. McGrath, "Five-year outcome study of deep vein thrombosis in the lower limbs," Journal of Vascular Surgery, vol. 40, no. 6, pp. 1184-1189, 2004.

[10] D. Imberti, C. Dall'asta, and M. G. Pierfranceschi, "Oral factor $\mathrm{Xa}$ inhibitors for thromboprophylaxis in major orthopedic surgery: a review," Internal and Emergency Medicine, vol. 4, no. 6, pp. 471-477, 2009.

[11] G. Agnelli and C. Becattini, "New oral anticoagulants: just a new therapeutic option or a real breakthrough?" Internal and Emergency Medicine, vol. 4, no. 6, pp. 455-457, 2009.

[12] V. V. Kakkar, T. P. Corrigan, and D. P. Fossard, "Prevention of fatal postoperative pulmonary embolism by low doses of heparin. An international multicentre trial," The Lancet, vol. 2, no. 7924, pp. 45-51, 1975.

[13] S. Sagar, D. Nairn, J. D. Stamatakis et al., "Efficacy of low-dose heparin in prevention of extensive deep-vein thrombosis in patients undergoing total-hip replacement," The Lancet, vol. 1, no. 7970, pp. 1151-1154, 1976.

[14] J. Hirsh, K. A. Bauer, M. B. Donati, M. Gould, M. M. Samama, and J. I. Weitz, "Parenteral anticoagulants: American College of Chest Physicians evidence-based clinical practice guidelines (8th edition)," Chest, vol. 133, no. 6, supplement, pp. 141S-159S, 2008.

[15] W. H. Geerts, J. A. Heit, G. P. Clagett et al., "Prevention of venous thromboembolism," Chest, vol. 119, no. 1, supplement, pp. 132S-175S, 2001.

[16] A. G. G. Turpie, "Oral, direct factor Xa inhibitors in development for the prevention and treatment of thromboembolic diseases," Arteriosclerosis, Thrombosis, and Vascular Biology, vol. 27, no. 6, pp. 1238-1247, 2007.

[17] M. R. Lassen, K. A. Bauer, B. I. Eriksson, and A. G. G. Turpie, "Postoperative fondaparinux versus preoperative enoxaparin for prevention of venous thromboembolism in elective hipreplacement surgery: a randomised double-blind comparison," The Lancet, vol. 359, no. 9319, pp. 1715-1720, 2002.

[18] K. A. Bauer, B. I. Eriksson, M. R. Lassen, and A. G. G. Turpie, "Fondaparinux compared with enoxaparin for the prevention of venous thromboembolism after elective major knee surgery," The New England Journal of Medicine, vol. 345, no. 18, pp. 1305-1310, 2001.

[19] A. Turpie, K. Bauer, B. Eriksson, and M. Lassen, "Efficacy and safety of fondaparinux in major orthopedic surgery according to the timing of its first administration," Thrombosis and Haemostasis, vol. 90, no. 2, pp. 364-366, 2003. 
[20] M. N. Levine, M. Gent, J. Hirsh et al., "Ardeparin (lowmolecular-weight heparin) vs graduated compression stockings for the prevention of venous thromboembolism: a randomized trial in patients undergoing knee surgery," Archives of Internal Medicine, vol. 156, no. 8, pp. 851-856, 1996.

[21] R. J. Friedman, A. S. Gallus, F. D. Cushner, G. FitzGerald, and F. A. Anderson Jr., "Physician compliance with guidelines for deep-vein thrombosis prevention in total hip and knee arthroplasty," Current Medical Research and Opinion, vol. 24, no. 1, pp. 87-97, 2008.

[22] A. T. Cohen, V. F. Tapson, J.-F. Bergmann et al., "Venous thromboembolism risk and prophylaxis in the acute hospital care setting (ENDORSE study): a multinational crosssectional study," The Lancet, vol. 371, no. 9610, pp. 387-394, 2008.

[23] J. Ansell, J. Hirsh, E. Hylek, A. Jacobson, M. Crowther, and G. Palareti, "Pharmacology and management of the vitamin K antagonists: American College of Chest Physicians evidencebased clinical practice guidelines (8th Edition)," Chest, vol. 133, no. 6, supplement, pp. 160S-198S, 2008.

[24] A. F. Shorr, E. A. Nutescu, E. Farrelly, R. Horblyuk, L. E. Happe, and M. Franklin, "Postdischarge oral versus injectable anticoagulation following major orthopedic surgery," Annals of Pharmacotherapy, vol. 42, no. 9, pp. 1222-1228, 2008.

[25] Pradaxa ${ }^{\circledR}$, "Summary of product characteristics," 2008, http:/ /www.pradaxa.com/Include/media/pdf/Pradaxa _ SPC _ EMEA.pdf.

[26] Xarelto ${ }^{\circledR}$, “Summary of product characteristics," 2009, http://www.xarelto.com/html/downloads / Xarelto_Summary_ of_Product_Characteristics_May2009.pdf.

[27] C. Frost, Z. Yu, S. Nepal, R. Mosqueda-Garcia, and A. Shenker, "Apixaban, an oral direct factor Xa inhibitor: singledose safety, pharmacokinetics and pharmacodynamics in healthy volunteers," Journal of Thrombosis and Haemostasis, vol. 5, supplement 1, 2007, Abstract no. P-M-665.

[28] C. E. Frost, S. Nepal, Y. Barrett, and F. LaCreta, "Effects of age and gender on the single-dose pharmacokinetics (PK) and pharmacodynamics (PD) of apixaban," in Proceedings of the 22nd congress of the International Society on Thrombosis and Haemostasis, Boston, Mass, USA, July 2009, Abstract no. PP-M0-407.

[29] N. Raghavan, C. E. Frost, Z. Yu et al., "Apixaban metabolism and pharmacokinetics after oral administration to humans," Drug Metabolism and Disposition, vol. 37, no. 1, pp. 74-81, 2009.

[30] C. Frost, L. Lee, L. Y. Li, S. Nepal, A. Shenker, and R. A. Reeves, "Apixaban does not affect the pharmacokinetics of digoxin," Journal of Clinical Pharmacology, vol. 47, no. 9, 2007, Abstract no. 60 .

[31] L. Wang, D. Zhang, N. Raghavan et al., "In vitro assessment of metabolic drug-drug interaction potential of apixaban through cytochrome p450 phenotyping, inhibition, and induction studies," Drug Metabolism and Disposition, vol. 38, no. 3, pp. 448-458, 2010.

[32] J. Carreiro and J. Ansell, "Apixaban, an oral direct factor Xa inhibitor: awaiting the verdict," Expert Opinion on Investigational Drugs, vol. 17, no. 12, pp. 1937-1945, 2008.

[33] M. R. Lassen, B. L. Davidson, A. Gallus, G. Pineo, J. Ansell, and D. Deitchman, "The efficacy and safety of apixaban, an oral, direct factor Xa inhibitor, as thromboprophylaxis in patients following total knee replacement," Journal of Thrombosis and Haemostasis, vol. 5, no. 12, pp. 2368-2375, 2007.
[34] M. R. Lassen, G. E. Raskob, A. Gallus, G. Pineo, D. Chen, and R. J. Portman, "Apixaban or enoxaparin for thromboprophylaxis after knee replacement," The New England Journal of Medicine, vol. 361, no. 6, pp. 594-604, 2009.

[35] M. R. Lassen, G. E. Raskob, A. Gallus, G. Pineo, D. Chen, and P. Hornick, "Apixaban versus enoxaparin for thromboprophylaxis after knee replacement (ADVANCE-2): a randomised double-blind trial," The Lancet, vol. 375, no. 9717, pp. 807-815, 2010.

[36] J. Stangier, "Clinical pharmacokinetics and pharmacodynamics of the oral direct thrombin inhibitor dabigatran etexilate," Clinical Pharmacokinetics, vol. 47, no. 5, pp. 285-295, 2008.

[37] J. Stangier, H. Stähle, K. Rathgen, and R. Fuhr, "Pharmacokinetics and pharmacodynamics of the direct oral thrombin inhibitor dabigatran in healthy elderly subjects," Clinical Pharmacokinetics, vol. 47, no. 1, pp. 47-59, 2008.

[38] S. Blech, T. Ebner, E. Ludwig-Schwellinger, J. Stangier, and W. Roth, "The metabolism and disposition of the oral direct thrombin inhibitor, dabigatran, in humans," Drug Metabolism and Disposition, vol. 36, no. 2, pp. 386-399, 2008.

[39] J. Stangier, B. I. Eriksson, O. E. Dahl et al., "Pharmacokinetic profile of the oral direct thrombin inhibitor dabigatran etexilate in healthy volunteers and patients undergoing total hip replacement," Journal of Clinical Pharmacology, vol. 45, no. 5, pp. 555-563, 2005.

[40] B. I. Eriksson, O. E. Dahl, L. Ahnfelt et al., "Dose escalating safety study of a new oral direct thrombin inhibitor, dabigatran etexilate, in patients undergoing total hip replacement: BISTRO I," Journal of Thrombosis and Haemostasis, vol. 2, no. 9, pp. 1573-1580, 2004.

[41] B. I. Eriksson, O. E. Dahl, H. R. Büller et al., "A new oral direct thrombin inhibitor, dabigatran etexilate, compared with enoxaparin for prevention of thromboembolic events following total hip or knee replacement: the BISTRO II randomized trial," Journal of Thrombosis and Haemostasis, vol. 3, no. 1, pp. 103-111, 2005.

[42] B. I. Eriksson, O. E. Dahl, N. Rosencher et al., "Oral dabigatran etexilate vs. subcutaneous enoxaparin for the prevention of venous thromboembolism after total knee replacement: the RE-MODEL randomized trial," Journal of Thrombosis and Haemostasis, vol. 5, no. 11, pp. 2178-2185, 2007.

[43] B. I. Eriksson and R. Friedman, "Dabigatran etexilate: pivotal trials for venous thromboembolism prophylaxis after hip or knee arthroplasty," Clinical and Applied Thrombosis/Hemostasis, vol. 15, pp. 25S-31S, 2009.

[44] The RE-MOBILIZE Writing Committee, "The oral thrombin inhibitor dabigatran etexilate vs the North American enoxaparin regimen for the prevention of venous thromboembolism after knee arthroplasty surgery," Journal of Arthroplasty, vol. 24, no. 1, pp. 1-9, 2009.

[45] B. I. Eriksson, O. E. Dahl, N. Rosencher et al., "Dabigatran etexilate versus enoxaparin for prevention of venous thromboembolism after total hip replacement: a randomised, double-blind, non-inferiority trial," The Lancet, vol. 370, no. 9591, pp. 949-956, 2007.

[46] S. E. Wolowacz, N. S. Roskell, J. M. Plumb, J. A. Caprini, and B. I. Eriksson, "Efficacy and safety of dabigatran etexilate for the prevention of venous thromboembolism following total hip or knee arthroplasty: a meta-analysis," Thrombosis and Haemostasis, vol. 101, no. 1, pp. 77-85, 2009.

[47] R. J. Friedman, O. E. Dahl, N. Rosencher et al., "Dabigatran versus enoxaparin for prevention of venous thromboembolism after hip or knee arthroplasty: a pooled analysis of three trials," Thrombosis Research. In press. 
[48] D. Kubitza, M. Becka, W. Mueck, and M. Zuehlsdorf, "The effect of extreme age, and gender, on the pharmacology and tolerability of rivaroxaban-an oral, direct factor Xa inhibitor," Blood, vol. 108, no. 11, 2006, Abstract no. 905.

[49] D. Kubitza, M. Becka, M. Zuehlsdorf, and W. Mueck, "Body weight has limited influence on the safety, tolerability, pharmacokinetics, or pharmacodynamics of rivaroxaban (BAY 59-7939) in healthy subjects," Journal of Clinical Pharmacology, vol. 47, no. 2, pp. 218-226, 2007.

[50] C. Weinz, T. Schwarz, D. Kubitza, W. Mueck, and D. Lang, "Metabolism and excretion of rivaroxaban, an oral, direct factor xa inhibitor, in rats, dogs, and humans," Drug Metabolism and Disposition, vol. 37, no. 5, pp. 1056-1064, 2009.

[51] D. Kubitza, M. Becka, W. Mueck, and M. Zuehlsdorf, "Rivaroxaban (BAY 59-7939)—an oral, direct factor Xa inhibitor-has no clinically relevant interaction with naproxen," British Journal of Clinical Pharmacology, vol. 63, no. 4, pp. 469-476, 2007.

[52] D. Kubitza, M. Becka, W. Mueck, and M. Zuehlsdorf, "Safety, tolerability, pharmacodynamics, and pharmacokinetics of rivaroxaban - an oral, direct factor Xa inhibitor-are not affected by aspirin," Journal of Clinical Pharmacology, vol. 46, no. 9, pp. 981-990, 2006.

[53] D. Kubitza, M. Becka, W. Mueck, and M. Zuehlsdorf, "Coadministration of rivaroxaban-a novel, oral, direct factor Xa inhibitor-and clopidogrel in healthy subjects," European Heart Journal, vol. 28, supplement 1, p. 189, 2007, Abstract no. P1272.

[54] D. Kubitza, M. Becka, and M. Zuehlsdorf, "No interaction between the novel, oral direct factor Xa inhibitor BAY 59-7939 and digoxin," Journal of Clinical Pharmacology, vol. 46, no. 6, p. 702, 2006, Abstract no. 11.

[55] B. I. Eriksson, L. C. Borris, O. E. Dahl et al., "Dose-escalation study of rivaroxaban (BAY 59-7939) — an oral, direct factor Xa inhibitor-for the prevention of venous thromboembolism in patients undergoing total hip replacement," Thrombosis Research, vol. 120, no. 5, pp. 685-693, 2007.

[56] B. I. Eriksson, L. Borris, O. E. Dahl et al., "Oral, direct factor Xa inhibition with BAY 59-7939 for the prevention of venous thromboembolism after total hip replacement," Journal of Thrombosis and Haemostasis, vol. 4, no. 1, pp. 121-128, 2006.

[57] B. I. Eriksson, L. C. Borris, O. E. Dahl et al., "A once-daily, oral, direct factor Xa inhibitor, rivaroxaban (BAY 59-7939), for thromboprophylaxis after total hip replacement," Circulation, vol. 114, no. 22, pp. 2374-2381, 2006.

[58] A. G. G. Turpie, W. D. Fisher, K. A. Bauer et al., "BAY 59-7939: an oral, direct factor Xa inhibitor for the prevention of venous thromboembolism in patients after total knee replacement. A phase II dose-ranging study," Journal of Thrombosis and Haemostasis, vol. 3, no. 11, pp. 2479-2486, 2005.

[59] B. I. Eriksson, L. C. Borris, R. J. Friedman et al., "Rivaroxaban versus enoxaparin for thromboprophylaxis after hip arthroplasty," The New England Journal of Medicine, vol. 358, no. 26, pp. 2765-2775, 2008.

[60] A. K. Kakkar, B. Brenner, O. E. Dahl et al., "Extended duration rivaroxaban versus short-term enoxaparin for the prevention of venous thromboembolism after total hip arthroplasty: a double-blind, randomised controlled trial," The Lancet, vol. 372, no. 9632, pp. 31-39, 2008.

[61] M. R. Lassen, W. Ageno, L. C. Borris et al., "Rivaroxaban versus enoxaparin for thromboprophylaxis after total knee arthroplasty," The New England Journal of Medicine, vol. 358, no. 26, pp. 2776-2786, 2008.
[62] A. G. G. Turpie, M. R. Lassen, B. L. Davidson et al., "Rivaroxaban versus enoxaparin for thromboprophylaxis after total knee arthroplasty (RECORD4): a randomised trial," The Lancet, vol. 373, no. 9676, pp. 1673-1680, 2009.

[63] B. I. Eriksson, A. K. Kakkar, and A. G. G. Turpie, "Oral rivaroxaban for the prevention of symptomatic venous thromboembolism after elective hip and knee replacement," Journal of Bone and Joint Surgery British, vol. 91, no. 5, pp. 636-644, 2009.

[64] A. G. G. Turpie, M. R. Lassen, A. K. Kakkar et al., "A pooled analysis of four pivotal studies of rivaroxaban for the prevention of venous thromboembolism after orthopaedic surgery: effect on symptomatic venous thromboembolism and death, and bleeding," Haematologica, vol. 94, supplement 2, p. 212, 2009.

[65] R. J. Friedman, A. G. G. Turpie, M. R. Lassen et al., "A pooled analysis of four pivotal studies of rivaroxaban for the prevention of venous thromboembolism after hip or knee arthroplasty," in Proceedings of the 122nd Annual Meeting of the American Orthopaedic Association, Bonita Springs, Fla, USA, June 2009, Abstract no. 314.

[66] R. H. Fitzgerald Jr., T. E. Spiro, A. A. Trowbridge et al., "Prevention of venous thromboembolic disease following primary total knee arthroplasty: a randomized, multicenter, open-label, parallel-group comparison of enoxaparin and warfarin," Journal of Bone and Joint Surgery American, vol. 83, no. 6, pp. 900-906, 2001.

[67] A. Squizzato, F. Dentali, L. Steidl, and W. Ageno, "New direct thrombin inhibitors," Internal and Emergency Medicine, vol. 4, no. 6, pp. 479-484, 2009. 


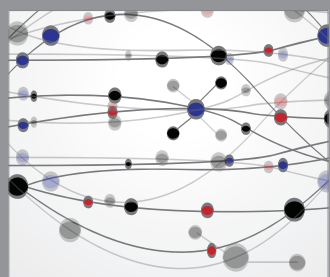

The Scientific World Journal
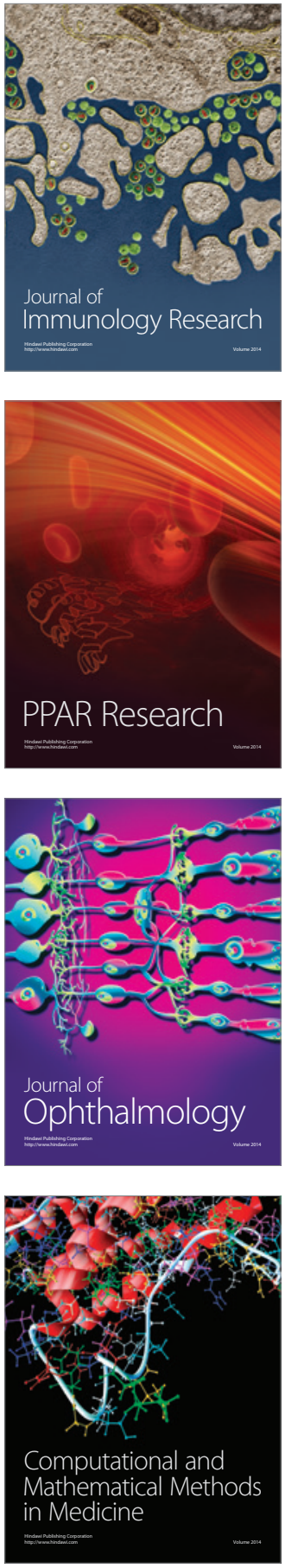

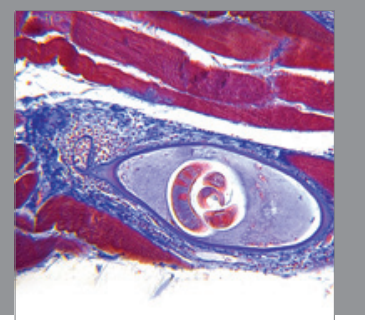

Gastroenterology

Research and Practice
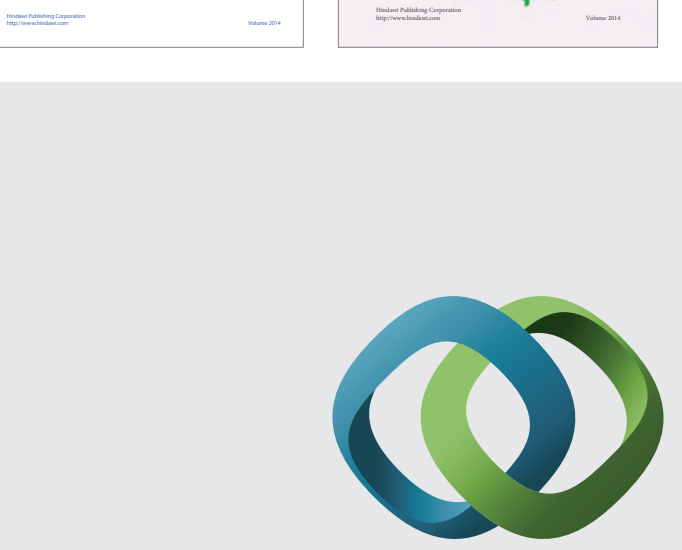

\section{Hindawi}

Submit your manuscripts at

http://www.hindawi.com
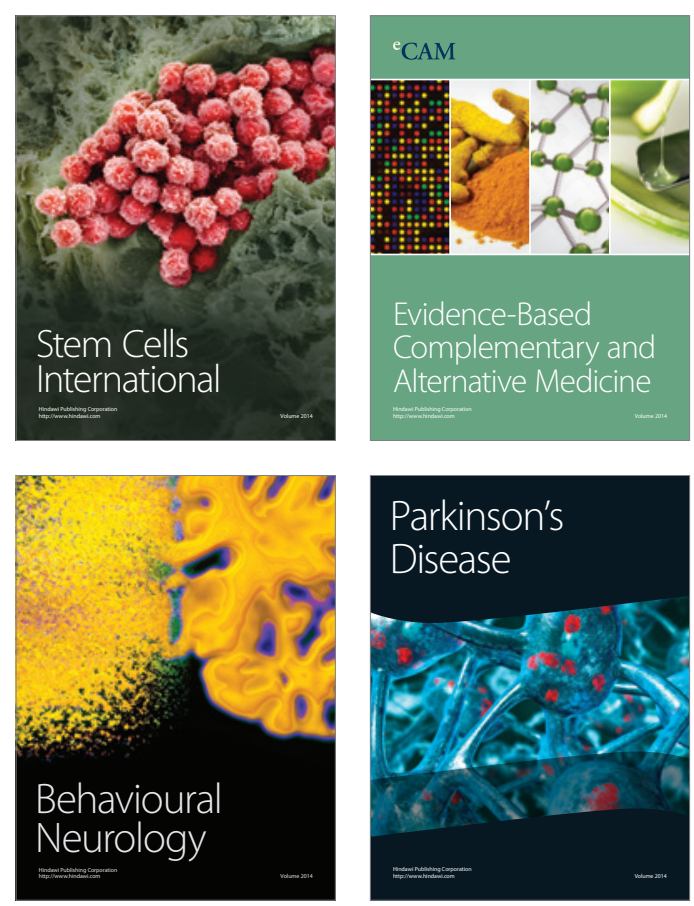

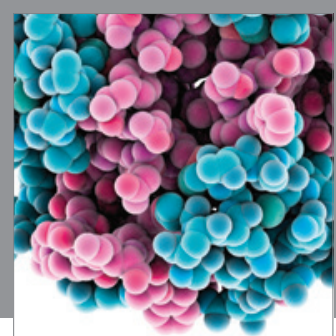

Journal of
Diabetes Research

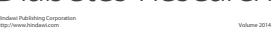

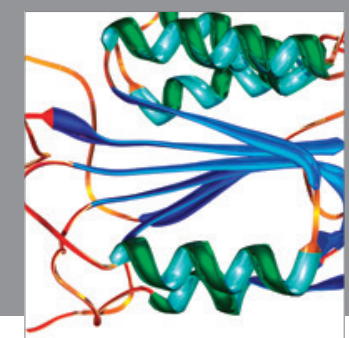

Disease Markers
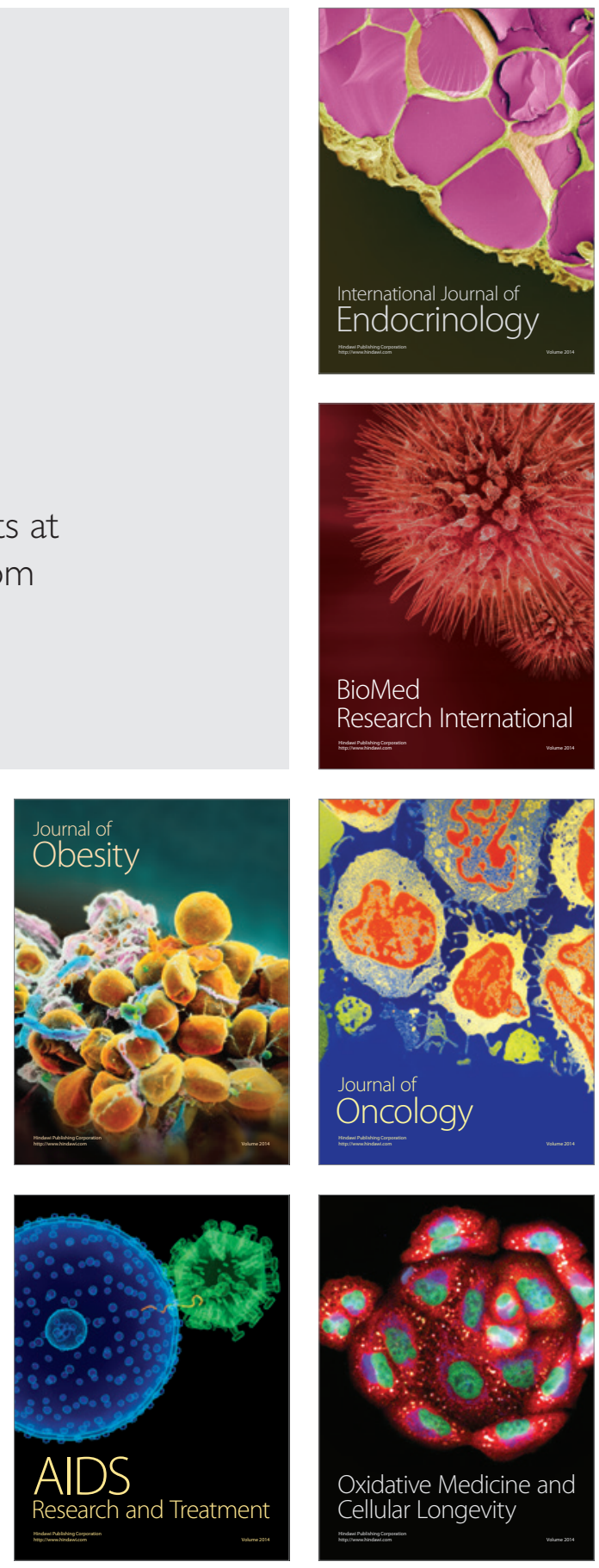